Supporting Information

\title{
Precise Perforation and Scalable Production of Si Particles from Low Grade Sources for High Performance Lithium Ion Battery Anodes
}

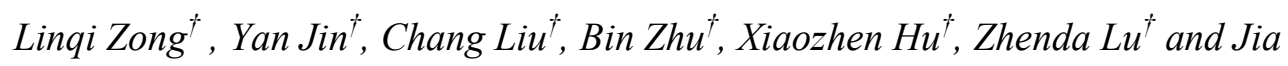
$Z h u^{* \prime}$

$\uparrow$ National Laboratory of Solid State Microstructures, College of Engineering and Applied Sciences and Collaborative Innovation Center of Advanced Microstructures, Nanjing University, Nanjing 210093, P. R. China.

*E-mail: jiazhu@nju.edu.cn (J. Z.)

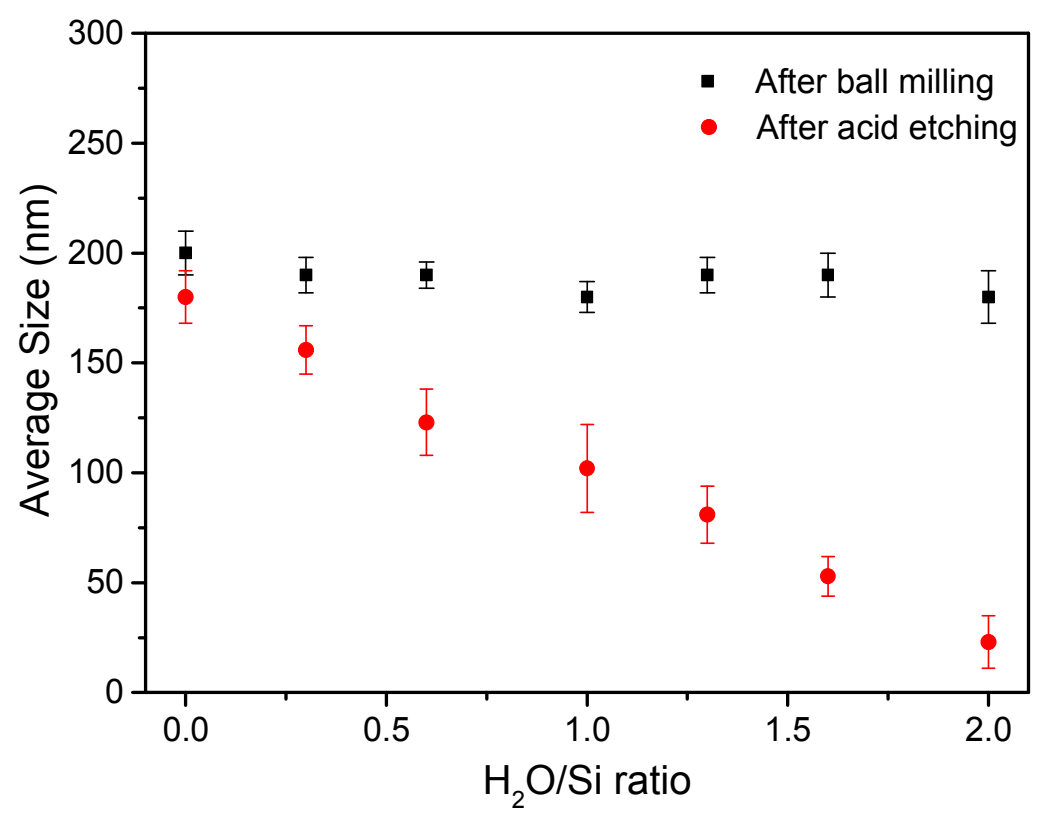

Figure S1. DLS test analysis of particles size after ball milling and acid etching. 


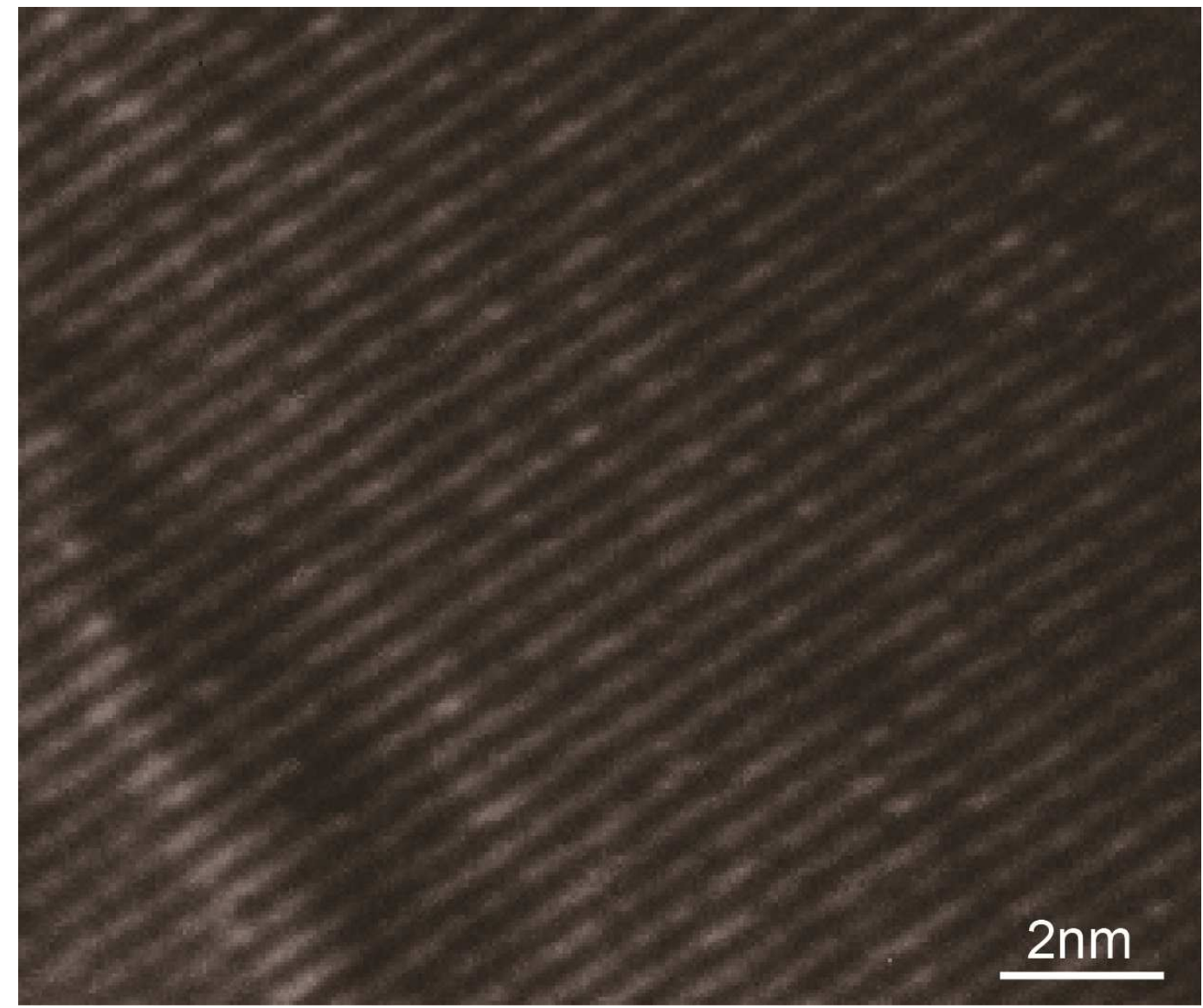

Figure S2. High resolution TEM image of porous silicon particles. 